\title{
Evaluación de una intervención educativa en el estilo de vida para prevenir prediabetes o diabetes tipo 2 en niños mexicanos
}

\section{Assessment of a lifestyle education intervention to prevent prediabetes or type 2 diabetes among mexican children}

\author{
Vanessa Mota Sanhua, ${ }^{\star}$ Adriana Reyes Camacho, ${ }^{\text {P Paulina Estefan Juárez, } § \text { Diana Martínez Castañeda, }, ~}$ \\ María Fernanda Guadalupe Calero Plaza,§ Blanca Velázquez Hernández"
}

Citar como: Mota SV, Reyes CA, Estefan JP, Martínez CD, Calero PMFG, Velázquez HB. Evaluación de una intervención educativa en el estilo de vida para prevenir prediabetes o diabetes tipo 2 en niños mexicanos. An Med (Mex). 2021; 66 (1): 25-35. https://dx.doi. org/10.35366/99486

\section{RESUMEN}

Introducción: La presencia de sobrepeso u obesidad es uno de los factores de riesgo modificables más importantes asociados con prediabetes y diabetes tipo 2 en todos los grupos de edad. En la actualidad, uno de cada tres niños y adolescentes mexicanos entre los seis y 19 años padece sobrepeso u obesidad. Los programas en el estilo de vida son prioritarios para impulsar el mantenimiento de un peso saludable y prevenir prediabetes y diabetes tipo 2. Objetivo: Evaluar el efecto de una intervención educativa en el estilo de vida para prevenir prediabetes o diabetes tipo 2 en niños mexicanos. Material y métodos: Piloto de un ensayo clínico controlado aleatorizado,

\section{ABSTRACT}

Introduction: Being overweight or obese, is one of the strongest modifiable risk factors associated with prediabetes and type 2 diabetes in all age groups. Currently, one out of three Mexican children and adolescents between six and 19 years old are overweight or obese. Lifestyle programs are prioritized to promote healthy weight maintenance and prevent prediabetes and type 2 diabetes. Objective: To assess a lifestyle education intervention to prevent prediabetes or type 2 diabetes among Mexican children. Material and methods: A clinical controlled trial designed as a randomized, single blind, parallel group, two arm pilot study. The sample comprised 26
* Doctora y Maestra en Ciencias, Licenciada en Nutrición y Ciencia de los Alimentos.

₹ Maestra en Nutrición Clínica. Clínica Brimex, Centro Médico ABC.

$\S$ Lic. en Nutrición y Ciencia de los alimentos. Clínica Brimex, Centro Médico ABC.

I Medicina y Maestra en Salud Pública. Clínica Brimex, Centro Médico ABC.

" Medicina, Especialidad en Patología Clínica y Maestra en Administración de Instituciones. Dirección Corporativa de Salud Incluyente y Educación, Centro Médico ABC.

\section{Correspondencia: Dra. Vanessa Mota Sanhua}

Clínica Brimex, Centro Médico ABC. Sur 136 Núm. 116,

Col. Las Américas, 01120. Alcaldía Álvaro Obregón, Ciudad de México,

Tel: 55 5230-8000, ext. 8803,

E-mail:vmotas@abchospital.com
Recibido: 02/10/2020. Aceptado: 26/03/2021.

\section{Abreviaturas:}

IMC = Índice de masa corporal.

$\mathrm{HbA1C}=$ Hemoglobina glicada .

HOMA-IR = Modelo homeostático para evaluar la resistencia a la insulina (Homeostatic Model Assessment-Insulin Resistance).

$\mathrm{HC}=$ Consumo de hidratos de carbono

OMS = Organización Mundial de la Salud.

$\mathrm{HC}=$ Hidratos de carbono 
ciego simple, con grupos paralelos, en dos brazos. La muestra está conformada por 26 niños de entre siete y 10 años, ambos sexos, con factores de riesgo de diabetes tipo 2 que se atienden en una clínica de primer nivel de atención en la Ciudad de México. La intervención tuvo una duración de cuatro meses. El grupo de intervención $(\mathrm{n}=12)$ recibió ocho sesiones grupales de educación en estilo de vida y cuatro consultas individuales de nutrición. El de control ( $\mathrm{n}=13)$, cuatro consultas individuales de nutrición y recomendaciones de rutina en el estilo de vida. Las variables principales fueron el índice de masa de corporal (IMC), porcentaje de grasa corporal, consumo de hidratos de carbono (g), HbA1c (hemoglobina glicada) y HOMA-IR (por las siglas en inglés de Homeostatic Model Assessment-Insulin Resistance). Los datos se analizaron con estadística descriptiva e inferencial (pruebas t de Student, t pareada y $\chi^{2}$ ). El nivel de significancia fue $p<0.05$. Resultados: Después de cuatro meses, la HbA1c disminuyó de forma estadísticamente significativa en ambos grupos, $0.32 \%$ en el grupo de estudio y $0.23 \%$ en el de control. El consumo de hidratos de carbono disminuyó en el grupo de estudio 20 gramos. El IMC y el porcentaje de grasa no tuvieron cambios en ningún grupo. Conclusión: Las intervenciones grupal e individual contribuyen a mejoras metabólicas. La primera promueve un menor consumo de hidratos de carbono.

Palabras clave: Diabetes mellitus tipo 2, estilo de vida, niño.

Nivel de evidencia: IV

\section{INTRODUCCIÓN}

En la actualidad, se ha declarado emergencia epidemiológica la diabetes tipo 2 y obesidad en diversos países, y México es uno de ellos. ${ }^{1}$ La prevalencia mundial de diabetes en el 2015 fue de uno de cada 11 y en el 2040 será uno de cada $10 .{ }^{1}$ En adultos mexicanos con diagnóstico previo, fue de uno de cada diez; ${ }^{2}$ en niños mexicanos no se tienen datos actuales. En este grupo sólo se cuenta con la referencia estadounidense del 4\%. ${ }^{3}$ En cuanto a prediabetes, en el 2013 la prevalencia mundial osciló entre 15 y $25 \% .{ }^{4}$ En adultos mexicanos aún es mayor, ${ }^{5}$ del $43.2 \%$, y en niños ${ }^{6}$ de entre seis y 12 años del seis al $12 \%$.

México ocupa el sexto lugar en el mundo entre los países con mayor número de personas con diabetes, esta enfermedad se encuentra entre las principales causas de morbi- y mortalidad nacional. ${ }^{7,8}$ Uno de los principales factores de riesgo de diabetes es el sobrepeso y la obesidad, ${ }^{1}$ además, se asocia con altos índices de intolerancia a la glucosa en todos los grupos de edad, lo cual define inicio de la patogenia de la enfermedad. ${ }^{3}$ Datos de la OCDE (Organización para la Cooperación y el Desarrollo Económico) reportan que México tiene el primer lugar en obesidad infantil en el ámbito mundial; en el 2018, uno de cada tres niños y adolescentes mexicanos la tuvieron. ${ }^{2}$ Un vas-
Mexican children, between seven and 10 years old, both sexes, with risk factors for type 2 diabetes, in a primary care clinic in Mexico City. The intervention was carried out in four months. Intervention group $(n=12)$ received 8 classes on lifestyle education and four nutrition consultations. Control group $(n=$ 13), four nutrition consultations and routine recommendations on lifestyle. Principal variables were Body Mass Index (BMI); fat mass percentage; carbohydrate consumption (g); HbA1c (Glycated hemoglobin) and, HOMA-IR (Homeostatic Model Assessment-Insulin Resistance). Data was analyzed through descriptive and inferential statistics ( $t$ Student test, $t$-paired test and $\chi^{2}$ test). Statistical significance was set at $p<0.05$. Results: After the intervention, HbA1c decreased, $0.32 \%$ and $0.23 \%$, in the intervention and control groups. Carbohydrate consumption decreased in $20 \mathrm{~g}$ in the intervention group. BMI and fat mass percentage did not change within any group. Conclusion: Group and individual interventions contribute to metabolic benefits. Group intervention promote carbohydrate consumption reduction.

Keywords: Diabetes mellitus type 2, life style, child.

Level of evidence: $I V$

to cuerpo de evidencia sustenta que los niños con sobrepeso u obesidad tienen riesgo de persistir con ella en la vida adulta. ${ }^{9}$ De acuerdo con guías para el tratamiento de diabetes, en niños con sobrepeso $\mathrm{u}$ obesidad es necesario el escrutinio de diabetes, ${ }^{10}$ en esta etapa, los niños aún no presentan cambios hormonales propios de los adolescentes que disminuyen la acción de insulina y aumentan la susceptibilidad de diabetes. ${ }^{3}$ Por ello, las intervenciones para prevenir o controlar el sobrepeso y la obesidad y sus comorbilidades, como la diabetes tipo 2 , pueden ser más efectivas.

La población infantil brinda oportunidad para combatir la obesidad y enfermedades como diabetes tipo 2, centrándose en cambios en el estilo de vida, aumento de la actividad física y disminución del consumo excesivo de alimentos de alto contenido energético y poco valor nutricional. ${ }^{11,12}$ En particular, la orientación alimentaria se enfoca en disminuir el consumo de alimentos que son fuente de hidratos de carbono simples o complejos con azúcar añadida, ${ }^{13}$ como consecuencia de la urbanización, el consumo de estos ha ido en aumento. ${ }^{14} \mathrm{En}$ la ENSANUT 2018, los hidratos de carbono simples o complejos con azúcar representan los porcentajes de consumo diario más altos de alimentos no recomendados en niños. ${ }^{2}$ 
Los proyectos, en su mayoría basados en los Programas de Prevención en Diabetes (Diabetes Prevention Program DPP, por sus siglas en inglés), han demostrado ser de bajo costo, seguros y efectivos, se basan en teorías de comportamiento para generar cambios. ${ }^{15}$ Los realizados en adultos en países como EUA, Australia, Reino Unido, Nueva Zelanda, Países Bajos, Finlandia, Canadá, Grecia, Israel, España y Japón incluyen estrategias de educación en nutrición, actividad física y cambios de conducta, ${ }^{16}$ los resultados muestran disminuciones en la incidencia de la enfermedad entre el 29 y $58 \%$, y pueden mantener el efecto hasta por 10 años. ${ }^{15}$ En niños hay menos investigaciones, algunas en México, Chile y EUA, ${ }^{17-20}$ sus resultados reflejan bases de evidencia limitada. ${ }^{21}$ Para nuestro conocimiento, no existen programas de intervención en el estilo de vida para promover la prevención de prediabetes o diabetes en niños mexicanos en los que evalúen conjuntamente variables metabólicas y del estado de nutrición.

En este contexto, y conforme a los lineamientos en México de la Academia Nacional de Medicina que emite un documento de postura sobre «Las acciones para enfrentar a la diabetes», surge la necesidad de diseñar e implementar protocolos de investigación en el primer nivel de atención del sistema de salud con enfoque en la población infantil. ${ }^{22}$

Objetivo: evaluar el efecto de una intervención educativa en el estilo de vida para prevenir prediabetes o diabetes tipo 2 en niños mexicanos.

\section{MATERIAL Y MÉTODOS}

Entre febrero y julio de 2016, se realizó un estudio piloto de un ensayo clínico controlado aleatorizado (ECA), ciego simple, con grupos paralelos, en dos brazos. Los criterios de inclusión fueron: niños entre siete y 10 años; afiliados a la clínica de primer nivel de atención del Centro Médico ABC, Ciudad de México; ambos sexos; bajo nivel socioeconómico; y con factores de riesgo de diabetes tipo 2, tales como: sobrepeso u obesidad, sedentarismo y/o tener un familiar en línea directa con diabetes tipo $2 .{ }^{3}$ Los niños que cumplieron los criterios quedaron enrolados en el estudio después de que su padre, madre y/o persona que ejerce la patria potestad otorgara consentimiento informado para su participación y que ellos aceptaran participar al conocer de lo que trataba el estudio. ${ }^{23}$ Se excluyeron niños con hipertensión arterial, prediabetes, diabetes tipo 1 o 2 , afección cardiaca $o$ alteraciones en tiroides, si durante la intervención se les diagnosticó alguna de dichas afecciones, quedaron eliminados, también aquéllos con $20 \%$ o más de inasistencia. El protocolo fue aprobado ante el Comité de Ética e Investigación del Centro Médico $\mathrm{ABC}$ con el número de registro $\mathrm{ABC}-1617$. Todos los participantes estuvieron acompañados de su cuidador primario. Cuando los niños completaron su participación en el estudio, continuaron su atención habitual en la clínica.

El grupo de intervención recibió ocho sesiones grupales de educación, con una hora de duración/ semana, en un aula en la clínica, el contenido de éstas se fundamentó en el estilo de vida saludable de dieta y actividad física. Se consideraron las guías y los lineamientos nacionales e internacionales para prevenir y tratar sobrepeso, obesidad, prediabetes y diabetes: 1) estándares de cuidados médicos en diabetes para niños y adolescentes de la Asociación Americana de Diabetes; ${ }^{24,25}$ 2) Norma Oficial Mexicana para la Atención a la Salud del Niño; ${ }^{26}$ 3) Norma Oficial Mexicana para la Atención a la Salud del Grupo Etario de 10 a 19 años de edad; ${ }^{27}$ 4) Norma Oficial Mexicana para la Prevención, Tratamiento y Control de la Diabetes Mellitus; ${ }^{28}$ y 5) Norma Oficial Mexicana para el Tratamiento Integral del Sobrepeso y la Obesidad. ${ }^{29}$ Las sesiones de educación se apoyaron en la teoría cognitiva social, que llama a construir la autoeficacia. ${ }^{30}$ Las estrategias educativas de esta teoría fueron la imitación de los pares, el entrenamiento para desarrollar habilidades y el establecimiento de objetivos. ${ }^{30}$ En la Tabla 1 se presentan los temas, recursos y estrategias cognitivas para las sesiones del grupo de intervención. De manera simultánea, los niños recibieron cuatro consultas individuales de nutrición, una al mes, y un plan de alimentación personalizado de acuerdo con las guías dietéticas del Instituto de Medicina ${ }^{31}$ y consejos para la actividad física de la Organización Mundial de la Salud (OMS) según sexo y edad. ${ }^{32}$ El grupo control recibió cuatro consultas mensuales de nutrición, una al mes, un plan de alimentación personalizado y consejos para la actividad física. Todo esto con las mismas características de las del grupo de intervención. También recomendaciones de rutina sobre estilo de vida para prevenir enfermedades crónicas, basadas en la normatividad mexicana para prevención, tratamiento y control de la diabetes mellitus. ${ }^{28}$ El estudio en ambos grupos tuvo una duración de cuatro meses. Se postuló que, después de ese tiempo, el grupo de intervención tuvo un efecto superior al control en las variables de tipo: 1) antropométrico y composición corporal: índice de masa corporal (IMC) y porcentaje de grasa; 2) dietético: consumo de hidratos de carbo- 
no (g); y 3) bioquímico: HbA1c (hemoglobina glicada) y HOMA-IR (por las siglas en inglés de Homeostatic Model Assessment-Insulin Resistance). Las sesiones educativas, las consultas de nutrición y la medición de variables se llevaron a cabo de manera estandarizada por nutriólogas previamente capacitadas.

\section{Definición de variables}

Antropométrica y composición corporal: la variable antropométrica fue el IMC y la de composición corporal fue el porcentaje de grasa corporal, cuando reflejan exceso de peso y grasa se relacionan con riesgo de prediabetes y diabetes tipo 2 . El IMC se estimó dividiendo peso ( $\mathrm{kg}$ ) entre talla al cuadrado (metros). Se usaron tablas de referencia por sexo y edad de la OMS para establecer el diagnóstico de nutrición por
IMC de los niños en categorías normal, sobrepeso u obesidad: ${ }^{33}$ 1) percentil entre 10 y 85 , normal; 2 ) percentil $\geq 85$ y $<95$, sobrepeso; y 3 ) percentil $\geq 95$, obesidad. El porcentaje de grasa corporal se categorizó en normal y alto con las tablas de referencia por sexo y edad: ${ }^{34}$ percentil $<90$ normal y percentil $\geq 90$ alto. El peso y el porcentaje de grasa corporal se obtuvieron del analizador de composición corporal Inbody 230 , solicitando al paciente acudir con al menos cuatro horas de ayuno. La talla se tomó por duplicado con el estadímetro SECA 213 y se calculó el promedio entre mediciones cuando la diferencia no era superior de $0.5 \mathrm{~mm}$. Cuando fue necesario, se tomó una tercera medición para calcular el promedio de las dos mediciones cuya diferencia era menor o igual a $0.5 \mathrm{~mm}$.

Dietética: la variable fue consumo de hidratos de carbono (HC) (g). Se evaluó la suma de los hidratos

Tabla 1: Temas, recursos y estrategias del grupo de intervención.

\begin{tabular}{|c|c|c|c|}
\hline Sesión & Tema & Recursos & Estrategia cognitiva social \\
\hline 1 & $\begin{array}{l}\text { Encuadre } \\
\text { Aplicación de cuestionario de frecuencia de consu- } \\
\text { mo de alimentos } \\
\text { Contrato conmigo mismo(a) y establecimiento de mis } \\
\text { objetivos }\end{array}$ & Cuestionarios & $\begin{array}{l}\text { Establecimiento de objetivos } \\
\text { Entrevista de motivación }\end{array}$ \\
\hline 3 & $\begin{array}{l}\text { Herramientas para la orientación alimentaria: } \\
\text { 1) Plato del bien comer } \\
\text { 2) Jarra del buen beber }\end{array}$ & $\begin{array}{l}\text { Poster del plato del bien co- } \\
\text { mer y la jarra del buen beber }\end{array}$ & $\begin{array}{l}\text { Desarrollo de habilidades y entrenamiento } \\
\text { Demostración y modelización } \\
\text { Control de estímulos }\end{array}$ \\
\hline 4 & $\begin{array}{l}\text { Los } 3 \text { grandes grupos: 1) verduras y frutas, 2) cerea- } \\
\text { les y leguminosas y 3) alimentos de origen animal }\end{array}$ & $\begin{array}{l}\text { Réplicas y empaques de } \\
\text { alimentos }\end{array}$ & $\begin{array}{l}\text { Refuerzo } \\
\text { Demostración y modelización } \\
\text { Desarrollo de habilidades y entrenamiento }\end{array}$ \\
\hline 6 & $\begin{array}{l}\text { Uso de alimentos equivalentes y de bajo índice } \\
\text { glucémico }\end{array}$ & $\begin{array}{l}\text { Imágenes de alimentos } \\
\text { equivalentes }\end{array}$ & $\begin{array}{l}\text { Control de estímulos } \\
\text { Demostración y modelización } \\
\text { Desarrollo de habilidades y entrenamiento }\end{array}$ \\
\hline 7 & Tipos de ejercicio y deportes & $\begin{array}{l}\text { Tapetes } \\
\text { Pelotas } \\
\text { Ligas } \\
\text { Aros }\end{array}$ & $\begin{array}{l}\text { Demostración y modelización } \\
\text { Desarrollo de habilidades y entrenamiento }\end{array}$ \\
\hline 8 & Actividad física: rutina de ejercicios & $\begin{array}{l}\text { Tapetes } \\
\text { Pelotas } \\
\text { Ligas } \\
\text { Aros }\end{array}$ & $\begin{array}{l}\text { Demostración y modelización } \\
\text { Desarrollo de habilidades y entrenamiento } \\
\text { Refuerzo }\end{array}$ \\
\hline
\end{tabular}




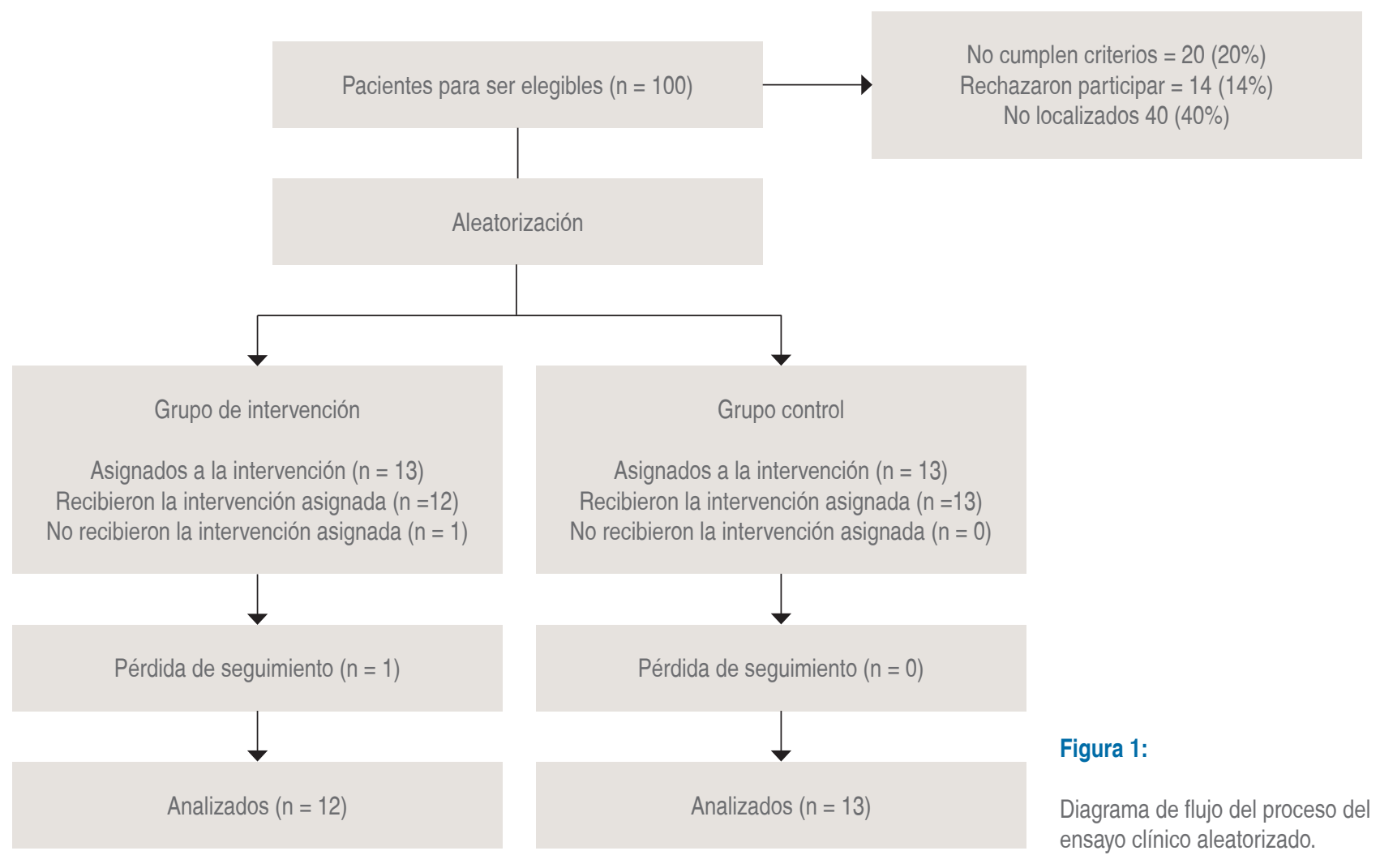

de carbonos, simples y complejos de los grupos de alimentos: cereales con y sin grasa, frutas y azúcares. El consumo de HC se evaluó con el cuestionario semicuantitativo de frecuencia de consumo de alimentos validado en población mexicana. Se contestó en privado por el niño y la madre, el padre y/o el cuidador primario. ${ }^{35}$ Las opciones de respuesta fueron consumo mensual, quincenal, semanal y diario. A partir de los alimentos reportados según su frecuencia, se sumó el contenido de $\mathrm{HC}$ de alimentos equivalentes de cereales con y sin grasa, frutas y azúcares. La variable quedó representada por el consumo diario de $\mathrm{HC}$ y se usó como variable cuantitativa continua para su análisis.

\section{Bioquímicos}

La HbA1c, la glucosa y la insulina en ayuno se determinaron a partir de muestras séricas en el laboratorio central del Centro Médico ABC después de 12 horas de ayuno del paciente. La glucosa y la insulina en ayuno se usaron para calcular HOMA-IR con la fórmula de Matthews DR y colaboradores: HOMA1$\mathrm{IR}=$ (insulina en ayuno $(\mathrm{mU} / \mathrm{l}) \times$ (glucosa sérica en ayuno $(\mathrm{mmol} / \mathrm{l}) / 22.5 .{ }^{36}$ Las variables de HbA1c y HO-
MA-IR se usaron como variables de tipo cuantitativo continua para el análisis de datos. No se emplearon puntos de corte por tratarse de pacientes sin prediabetes o diabetes tipo 2 .

El tamaño de la muestra fue estimado por diferencia de proporciones, con datos de referencia del estudio de Knowler y su equipo y se agregó el $15 \%$ de pérdidas. ${ }^{37}$ En total, se calcularon 56 pacientes por grupo. La asignación a los grupos se hizo por aleatorización simple en Excel con lista de aleatorización. La investigadora principal llevó a cabo el reclutamiento de los pacientes y la asignación aleatoria. Se mantuvo el ciego simple en los participantes, quienes desconocían las estrategias que se compararon en el estudio. Esto disminuyó el sesgo de respuestas motivadas por el tipo de intervención.

Todos los datos de los pacientes se capturaron en una hoja de cálculo de Excel y se exportaron al SPSS (Statistical Package for Social Sciences) versión 22.0 para realizar el análisis estadístico. En el descriptivo se obtuvo promedio y desviación estándar de las variables cuantitativas, y frecuencias y proporciones de las cualitativas. Se emplearon pruebas de $t$ de Student y $\chi^{2}$ para muestras independientes para evaluar que los grupos fueran homogéneos antes de 
la intervención, y también para evaluar la efectividad de los tratamientos entre grupos. La prueba de $t$ de Student para muestras dependientes se usó para comparar el antes y el después de cada grupo, el nivel de significancia fue de $\mathrm{p}<0.05$.

\section{RESULTADOS}

La población de la clínica con niños entre siete y 10 años fue de 121 niños. De ellos, 100 cumplieron los criterios de selección, 21 no cumplía porque no tuvieron ninguno de los factores de riesgo de diabetes tipo 2. Los elegibles que acudieron a la entrevista informativa junto con su padre, madre o cuidador primario fueron 26 niños. De los pacientes que no entraron al estudio, 20\% (20) tenía prediabetes, $14 \%$ (14) no aceptó participar y el $40 \%$ (40) no se contactaron. Los 26 niños quedaron dentro del estudio cuando el padre, madre o tutor firmaron la carta de consenti- miento informado y el niño el consentimiento. Cada grupo se conformó con 13 pacientes asignados de manera aleatoria. Durante el estudio, un paciente del grupo de intervención decidió abandonar el programa por cuestiones personales (Figura 1).

En la Tabla 2 se presentan las características de los pacientes de ambos grupos previo a la intervención. El $41.7 \%$ del grupo de estudio y el $23.1 \%$ del control fueron del sexo femenino. La media de edad fue de ocho años en los dos grupos. En promedio, ambos presentaron 6.7 y 8.7 factores de riesgo para prediabetes o diabetes tipo 2, los más frecuentes fueron sedentarismo, sobrepeso u obesidad y antecedentes familiares de diabetes tipo 2. El porcentaje de grasa alto estuvo en el $100 \%$ del grupo de estudio y el $77 \%$ del grupo control. No hay diferencias estadísticamente significativas entre grupos en ninguna de las variables.

Antes y después, en el grupo de estudio, se encontraron disminuciones estadísticamente significativas

Tabla 2: Características de los pacientes de grupos de estudio y control, antes de la intervención.

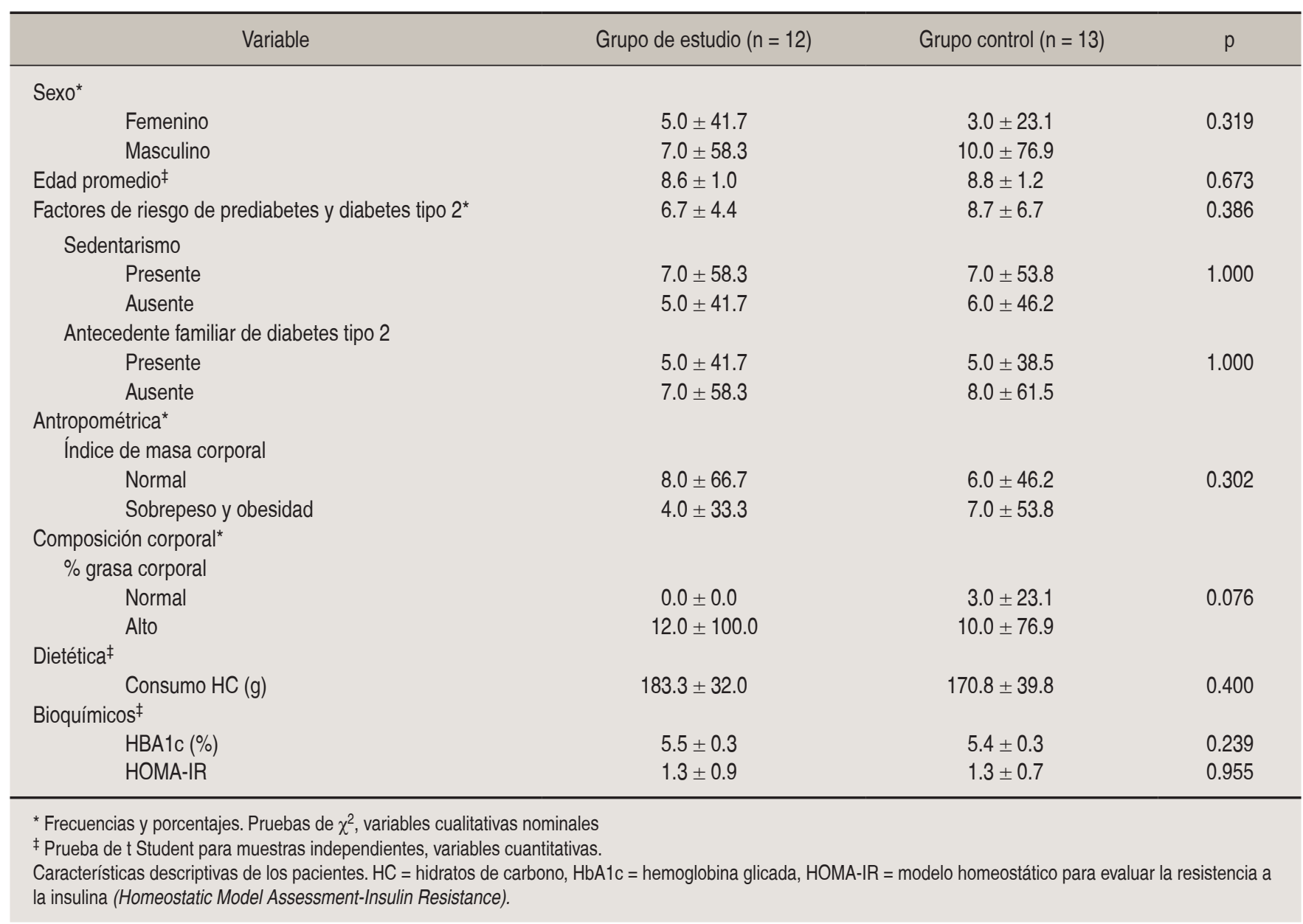


Tabla 3: Comparación antes y después en el grupo de estudio.

\begin{tabular}{|c|c|c|c|}
\hline \multirow[b]{2}{*}{ Variable } & \multicolumn{3}{|c|}{ Grupo de estudio $(n=12)$} \\
\hline & Antes & Después & $p$ \\
\hline \multicolumn{4}{|l|}{ Antropométrica* } \\
\hline \multicolumn{4}{|l|}{ Índice de masas corporal } \\
\hline Normal & $8.0 \pm 66.7$ & $8.0 \pm 66.7$ & 1.000 \\
\hline \multicolumn{4}{|l|}{ Composición corporal ${ }^{*}$} \\
\hline \multicolumn{4}{|l|}{$\%$ de grasa corporal } \\
\hline Normal & $0.0 \pm 0.0$ & $0.0 \pm 0.0$ & No se calcula \\
\hline Alto & $12.0 \pm 100.0$ & $12.0 \pm 100.0$ & \\
\hline \multicolumn{4}{|l|}{ Dietética $^{\ddagger}$} \\
\hline Consumo $\mathrm{HC}(\mathrm{g})$ & $183.3 \pm 32.0$ & $163.3 \pm 36.7$ & 0.029 \\
\hline \multicolumn{4}{|l|}{ Bioquímicos ${ }^{\ddagger}$} \\
\hline $\begin{array}{l}\text { * Frecuencias y proporciones, prueba de } \\
\text { Comparación antes y después de la inter } \\
\mathrm{HC}=\text { hidratos de carbono, } \mathrm{HbA} 1 \mathrm{C}=\text { hemc } \\
\text { Insulin Resistance). }\end{array}$ & omeostático para e & istencia a la insulin & Model Assessmen \\
\hline
\end{tabular}

en la HbA1c, del $0.32 \%$ ( $p=0.002$ ) y en el de consumo de HC, 20 gramos, $(p=0.029)$. No se observaron cambios en las variables del estado de nutrición ( $T a$ bla 3).

Antes y después, en el grupo control, se encontró disminución estadísticamente significativa en la HbA1c de 0.23\% ( $\mathrm{p}=0.011$ ). El HOMA-IR aumentó $0.3(\mathrm{p}=0.01)$. No hubo cambios en las variables del estado de nutrición (Tabla 4).

Después de la intervención, en el grupo de estudio el HOMA-IR es menor que el del control, pero no de manera estadísticamente significativa (Tabla 5).

\section{DISCUSIÓN}

El objetivo en este estudio piloto fue evaluar una intervención de educación en el estilo de vida para prevenir prediabetes o diabetes tipo 2 en niños. Hay suficiente evidencia que sustenta que un estilo de vida saludable reduce las tasas de incidencia de éstas en todos los grupos de edad. ${ }^{15,16,38}$

La educación grupal en el estilo de vida para prevenir prediabetes o diabetes tipo 2 se comparó contra la maniobra individual de rutina, después de cuatro meses, se vieron disminuciones de la $\mathrm{HbA} 1 \mathrm{c}$ en el grupo de intervención y el de control. De acuerdo con la revisión sistemática de Mancipe y colaborado- res, no hay estudios mexicanos en el estilo de vida en niños en edad escolar en los que se midan cambios metabólicos en HbA1c y HOMA-IR. ${ }^{20}$ Se realizó una investigación en adolescentes latinoamericanos por Soltero y su equipo, que consistió en una intervención durante tres meses, en donde se comparó contra grupo control. En el grupo del estilo de vida, la sensibilidad a la insulina mejoró; sin embargo, después de 12 meses, ésta no se mantuvo significativa. ${ }^{19} \mathrm{Se}$ sugiere continuar líneas de investigación similares para estandarizar protocolos de prevención de prediabetes y diabetes tipo 2 en niños, y evaluar su impacto con indicadores metabólicos.

En nuestro estudio, después de la intervención, las variables del IMC y el porcentaje de grasa corporal se mantuvieron sin cambios en ambos grupos. En estudios similares, sí se reportan beneficios en el estado de nutrición, por ejemplo, en el de Soltero y su grupo, adolescentes latinoamericanos con obesidad recibieron educación en el estilo de vida con sesiones sobre cambio de conducta, durante tres meses de tratamiento (tres días a la semana). En los resultados a los tres y 12 meses se observaron disminuciones en la masa grasa $(\mathrm{kg})$ : -2.52 y -3.36 y el IMC $\left(\mathrm{kg} / \mathrm{m}^{2}\right)$ : -1.02 y $-1.21{ }^{19}$ En la revisión sistemática de Mancipe y colaboradores, que incluye 17 estudios latinoamericanos, en sólo dos se vieron disminuciones y fue- 
ron en el IMC, de: -0.9 y $-1 \mathrm{~kg} / \mathrm{m}^{2} .{ }^{20}$ En el estudio de Elizondo y su equipo, en 96 niños mexicanos con sobrepeso u obesidad entre seis y 12 años se otorgaron 13 consultas de nutrición (cada tres semanas) a los niños y sus padres durante 10 meses, ${ }^{17}$ en los resultados 57\% de los niños disminuyeron su IMC y el porcentaje de grasa bajó $2.4 .{ }^{17}$ De acuerdo con estas evidencias, se sugiere que la mayor frecuencia y duración de las sesiones promueven disminuciones de grasa corporal e IMC. ${ }^{38}$ En el presente estudio se otorgó una sesión grupal por semana y una consulta de nutrición al mes durante cuatro meses. En los estudios con disminuciones de peso y grasa, la frecuencia de las sesiones fue de tres veces por semana y la duración de 10 meses.

Con base en estos resultados, se puede explicar que el beneficio metabólico fue independiente de las disminuciones en el peso. Será necesario realizar estudios con mayor tiempo de seguimiento para evaluar la mejora metabólica en la $\mathrm{HbA} 1 \mathrm{C}$ con respecto al peso corporal.

En la intervención grupal, se observó disminución significativa en el consumo de HC después de cuatro meses. Esto pudo explicarse por los conocimientos adquiridos en las sesiones grupales referente al contenido de $\mathrm{HC}$ en alimentos y bebidas, además del plan de alimentación personalizado. El contenido de la información se basó en la normatividad mexicana del plato del bien comer y la jarra del buen beber. ${ }^{13}$ En estudios latinoamericanos similares, cuyo objetivo fue determinar la efectividad de educación para prevenir sobrepeso y obesidad en niños de seis a 17 años, la evaluación del consumo de alimentos fue heterogénea; cada estudio utilizó un instrumento de medición diferente, ${ }^{20}$ en algunos se reportaron disminuciones en el consumo de energía total en kilocalorías, mientras que en otros disminuyó el consumo de alimentos no saludables. Los resultados han sido inconsistentes. ${ }^{20}$ Estas características limitan las comparaciones con nuestros resultados.

En la muestra de este estudio, los factores de riesgo más frecuentes para prediabetes y diabetes tipo 2 fueron sedentarismo (56\%) y sobrepeso u obesidad (44\%). Las cifras son parecidas a las de la ENSANUT 2016 en niños de la misma edad: el 82.8\% de los mexicanos fueron clasificados físicamente inactivos y el $35.6 \%$ con sobrepeso u obesidad. ${ }^{39} \mathrm{El}$ mayor porcentaje de niños con sedentarismo pudo deberse al instrumento de medición, que fue un cuestionario de actividad física; mientras que en esta investigación se hizo con una pregunta abierta sobre si realizaba actividad física, por lo que pudo haber existido un subregistro. Se sugiere realizar mediciones del nivel

Tabla 4: Comparación antes y después del grupo control.

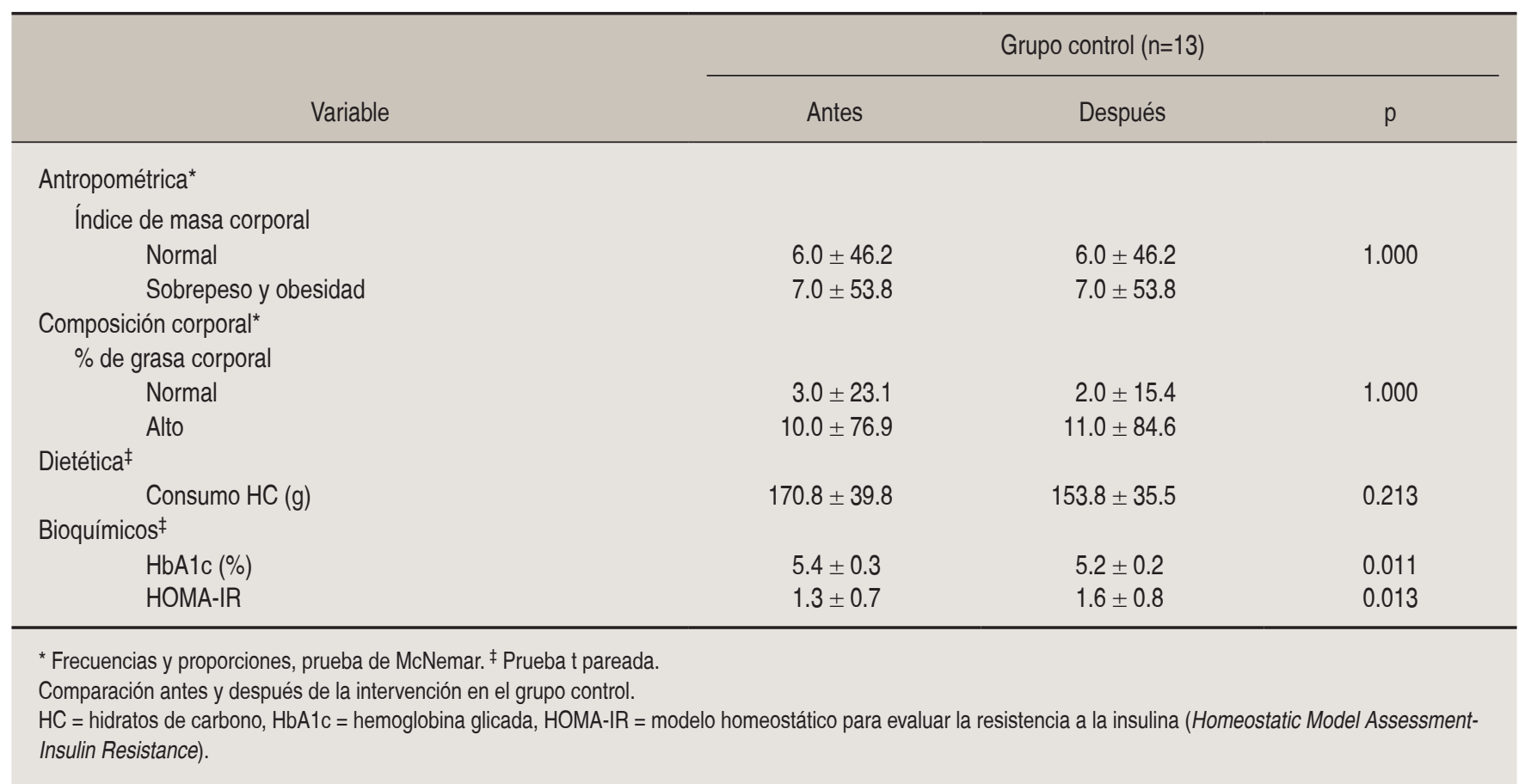


Tabla 5: Comparación entre el grupo de estudio y control después de la intervención.

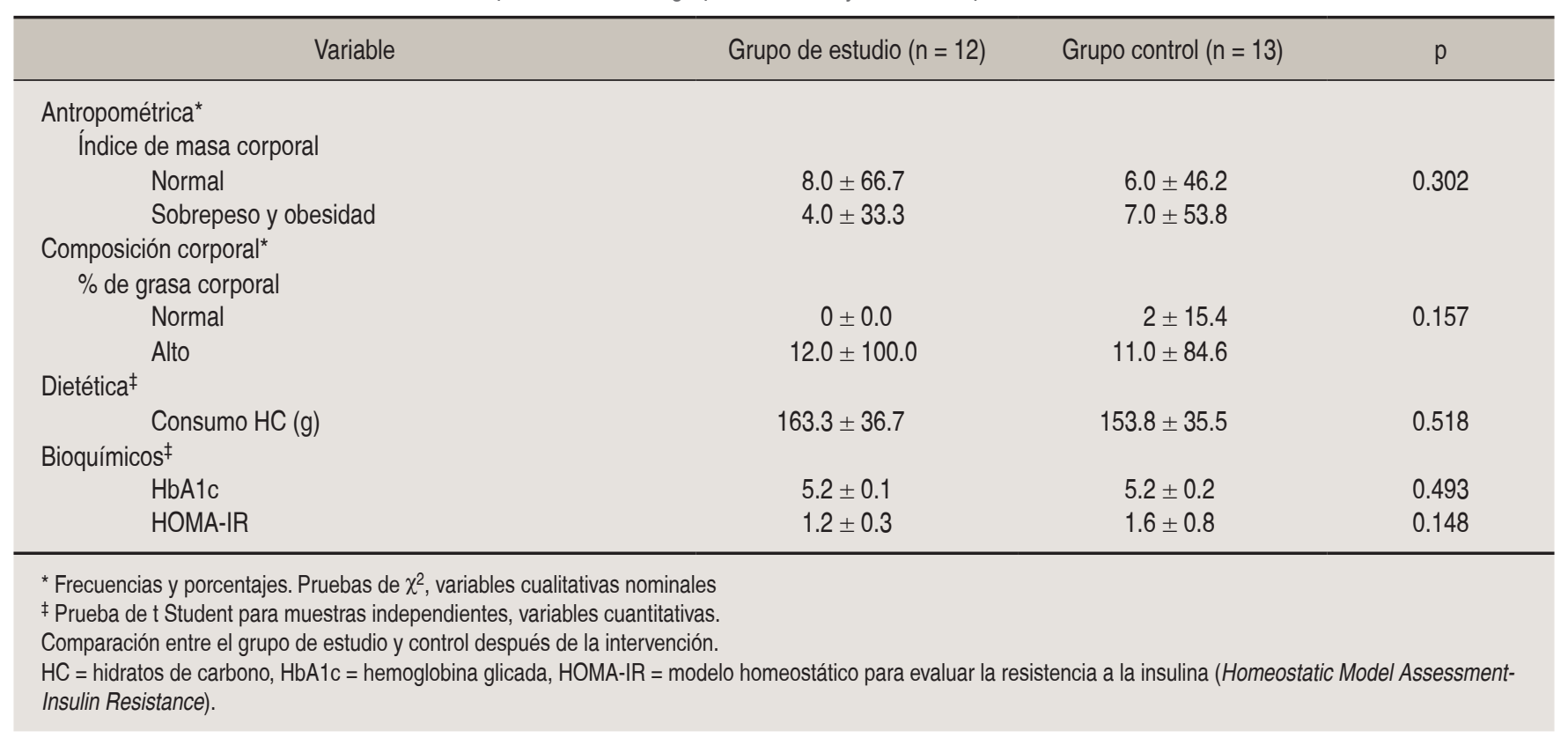

de actividad física con el instrumento empleado en las encuestas nacionales. De ese modo, los resultados de investigaciones podrán compararse con los de las encuestas. La prevalencia de sobrepeso u obesidad en nuestra muestra fue más alta a la de la encuesta de 2018, posiblemente porque se trata de pacientes en una clínica. ${ }^{2}$ A pesar de las diferencias de los factores de riesgo entre las poblaciones, las acciones preventivas de educación para la salud son necesarias a gran escala, de manera grupal e individual.

Al final de la intervención no se observaron diferencias significativas entre grupos para ninguna de las variables del estudio, es decir, ninguna intervención fue mejor que otra. Aquí se explican limitaciones y recomendaciones para estudios futuros.

Entre las limitaciones del estudio, hay que señalar el tamaño de muestra reducido, se completó el $22.3 \%$ de la calculada, por ello, los resultados tienen generalización (validez externa) limitada, y únicamente pueden tomarse como resultados de un estudio piloto. Una de las razones del pequeño tamaño de la muestra fue porque se tuvo dificultad en la invitación de casos a participar, la cual fue vía telefónica y en algunos pacientes no se contó con datos de contactos completos y/o actualizados. Será necesario desarrollar otras alternativas en las estrategias de invitación, por ejemplo, mediante redes sociales, correo electrónico o campañas en centros escolares. El propósito de realizar el estudio sólo en niños de bajo nivel socioeconómico fue por la estrecha relación entre el bajo nivel socioeconómico y la malnutrición. En este sentido, es prioridad estudiar los grupos más vulnerables. Quizá más adelante sea necesario analizar la efectividad de las intervenciones por nivel socioeconómico.

Algunas recomendaciones para futuros estudios son: 1) comparar mediciones de sensibilidad a la insulina, por ejemplo, una prueba sugerida para este grupo de edad es el Quantose-IR; ${ }^{40}$ 2) medir con instrumentos validados actividad física y consumo de hidratos de carbono en alimentos y bebidas, clasificados por su índice glucémico; 3) al tratarse de menores de edad, y conforme a las guías para el tratamiento de diabetes, ${ }^{25}$ diseñar intervenciones grupales o individuales, incluyendo al cuidador primario para facilitar la integración de los conocimientos en las rutinas del hogar; 4) ampliar los canales de comunicación con entrega de información a distancia para incluir y reforzar los estilos de vida saludables en más pacientes; 5) incluir mediciones antropométricas y bioquímicas de los padres de los niños para evaluar el componente hereditario en las variables de los niños.

\section{CONCLUSIÓN}

En este estudio se demuestra que la educación en el estilo de vida, grupal e individual, disminuye la 
HbA1c. Dicha mejoría se observa en ambos grupos sin presentarse disminuciones en IMC o grasa corporal. La disminución del consumo de HC se observó en la intervención grupal.

Los resultados apoyan que las intervenciones en el estilo de vida contribuyen en la prevención de prediabetes y diabetes tipo 2 . Se requieren otros estudios en niños con mayor tamaño de muestra y más seguimiento. Se sugiere extender de manera rutinaria el uso de indicadores metabólicos en niños con riesgo de desarrollar prediabetes y diabetes tipo 2 con el objeto de evaluar los efectos de las intervenciones educativas en el estilo de vida.

\section{REFERENCIAS}

1. Federación Internacional de Diabetes (FID). Atlas de la Diabetes de la Federación Internacional de Diabetes. Séptima Edición. International Diabetes Federación; 2015.

2. Instituto Nacional de Salud Pública. Presentación de resultados: Encuesta Nacional de Salud Pública 2018. México: Salud Pública de México-INEGI-Secretaría de Salud; 2018.

3. Frenk P, Márquez E. Diabetes mellitus tipo 2 en niños y adolescentes. Med Int Mex. 2010; 26 (1): 36-47.

4. Rosas J, Caballero A, Brito G, García H, Costa J, Lyra R et al. Consenso de Prediabetes. Documento de posición de la Asociación Latinoamericana de Diabetes (ALAD). Rev ALAD. 2017; 7: 184-202.

5. Guerrero F, Rodríguez M, Pérez R, Sánchez M, González M, Martínez E et al. Prediabetes and its Relationship with Obesity in Mexican Adults: The Mexican Diabetes Prevention (MexDiab) Study. Metabolic Syndr Relat Disord. 2008; 6 (1): 15-23.

6. Ávila A, Galindo C, Juárez L, Osorio M. Metabolic syndrome in children aged 6 to 12 years with obesity in public schools of seven municipalities in the state of Mexico. Salud Pública Mex. 2018; 60 (4): 395-403.

7. Dirección General de Epidemiología. Anuario de Morbilidad 1984-2019. Gobierno de México; 2019.

8. Federación Internacional de Diabetes (FID). Atlas de la Diabetes de la Federación Internacional de Diabetes. Sexta Edición. International Diabetes Federación; 2013.

9. Clinical Practice Guideline for the Prevention and Treatment of Childhood and Juvenile Obesity. Clinical Practice Guidelines in the Spanish National Healthcare System Ministry for Health and Social Policy. Spain: Ministry for Science and Innovation; 2009.

10. American Diabetes Association. 13. Children and adolescents: standards of medical care in diabetes-2019. Diabetes Care. 2018; 42 (Suppl 1): S148-S64.

11. Martínez A, Trescastro EM. Actividades de educación alimentaria y nutricional en escolares de 3o de primaria en el Colegio Público "La Serranica" de Aspe (Alicante): experiencia piloto. Rev Esp Nutr Hum Diet. 2016; 20 (2): 97-103.

12. Tárraga J, Tárraga M, Panisello J, Rosich N, Castell E, Carbayo J. Resultados de una intervención motivacional con niños obesos o con sobrepeso y sus familias: estudio piloto. Rev Esp Nutr Hum Diet. 2017; 21 (4): 313-319.

13. Secretaría de Salud, Norma Oficial Mexicana NOM-043SSA2-2005, Servicios básicos de salud. Promoción y educación para la salud en materia alimentaria. Criterios para brindar orientación. Diario Oficial de la Federación, 23 de enero de 2006.

14. Pérez O, Nazar A, Salvatierra B, Pérez-Gil S, Rodríguez L, Castillo T et al. Frecuencia del consumo de alimentos industrializados modernos en la dieta habitual de comunidades mayas de Yucatán, México. Estud Soc. 2012; 20 (39): 155-184.

15. Helitzer D, Bobo A, Thompson J, Fluder S. Development of a planning and evaluation methodology for assessing the contribution of theory to a diabetes prevention lifestyle intervention. Health Promot Pract. 2008; 9 (4): 404-414.

16. Galaviz K, Weber M, Straus A, Haw J, Narayan K, Ali M. Global diabetes prevention interventions: a systematic review and network meta-analysis of the real-world impact on incidence, weight, and glucose. Diabetes Care. 2018; 41 (7): 1526-1534.

17. Elizondo L, Gutierrez N, Moreno D, Martínez U, Tamargo D, Treviño M. School-based individualised lifestyle intervention decreases obesity and the metabolic syndrome in mexican children. J Hum Nutr Diet. 2013; 26 (Suppl 1): 82-89.

18. Fernández L, Leyton B, Kain J, Vio del Río F. Evaluación de una intervención educativa para la prevención de la obesidad infantil en escuelas básicas de Chile. Nutr Hosp. 2013; 28 (3): 1156-1164.

19. Soltero EG, Olson ML, Williams AN, Konopken YP, Castro FG, Arcoleo KJ et al. Effects of a community-based diabetes prevention program for latino youth with obesity: a randomized controlled trial. Obesity (Silver Spring). 2018; 26 (12): 1856-1865.

20. Mancipe J, García S, Correa J, Meneses J, González E, Schmidt J. Efectividad de las intervenciones educativas realizadas en América Latina para la prevención sobrepeso y obesidad infantil en niños escolares de 6 a 17 años: una revisión sistemática. Nutr Hosp. 2015; 31 (1): 102-114.

21. Haemer M, Grow H, Fernandez C, Lukasiewicz G, Rhodes E, Shaffer L et al. Addressing prediabetes in childhood obesity treatment programs: support from research and current practice. Child Obes. 2014; 10 (4): 292-303.

22. Aguilar SC, Hernández JS, Hernández AM, Hernández AJ. Acciones para enfrentar a la diabetes. México: Academia Nacional de Medicina; 2015.

23. Reglamento de la Ley General de Salud en Materia de Investigación para la Salud [Internet]. México: Diario Oficial de la Federación; 2019.

24. Classification and diagnosis of diabetes: standards of medical care in diabetes-2016. Diabetes Care. 2016; 39 (1): S13-S22. Diabetes Care. 2016; 39 (9): 1653. doi: 10.2337/dc16-er09. Erratum for: Diabetes Care. 2016; 39 (Suppl 1): S13-22.

25. American Diabetes Association. 11 Children and adolescents: standards of medical care in diabetes-2016. Diabetes Care. 2016; 39 (1): S86-S93.

26. Secretaría de Salud, Norma Oficial Mexicana NOM-031SSA2-1999, Para la atención a la salud del niño. Diario Oficial de la Federación, 9 de junio de 2000.

27. Secretaría de Salud, Norma Oficial Mexicana NOM-047SSA2-2015, Para la atención a la salud del grupo etario de 10 a19 años de edad. Diario Oficial de la Federación, 20 de febrero de 2015.

28. Secretaría de Salud, Norma Oficial Mexicana NOM-015SSA2-2010, Para la prevención, tratamiento y control de la diabetes mellitus. Diario Oficial de la Federación, 23 de junio de 2009 .

29. Secretaría de Salud, Norma Oficial Mexicana NOM-008SSA3-2010, Para el tratamiento integral del sobrepeso y la obesidad. Diario Oficial de la Federación, 7 de julio de 2010. 
30. Spahn J, Reeves R, Keim K, Laquatra I, Kellogg M, Jortberg B et al. State of the evidence regarding behavior change theories and strategies in nutrition counseling to facilitate health and food behavior change. J Am Diet Associ. 2010; 110 (6): 879891.

31. U.S. Department of Health and Human Services and U.S. Department of Agriculture. 2015-2020 Dietary Guidelines for Americans. 8th ed. U.S. Department of Health and Human Services and U.S. Department of Agriculture. 2015.

32. Global Strategy on Diet, Physical Activity and Health. Recommended levels of physical activity for children aged 5 17 years. Ginebra: Global Strategy on Diet, Physical Activity and Health. 2019.

33. World Health Organization (WHO). Growth reference data for 5-19 years. Ginebra: WHO, 2020.

34. Laurson K, Eisenmann J, Welk G. Body fat percentile curves for U.S. children and adolescents. Am J Prev Med. 2011; 41 (4): S87-S92.

35. Denova E, Ramírez I, Rodríguez S, Jiménez A, Shamah T, Rivera J. Validity of a food frequency questionnaire to assess food intake in Mexican adolescent and adult population. Salud Pública de México. 2016; 58 (6): 617-628.

36. Wallace TM, Levy JC, Mathews DR. Use and abuse of HOMA modeling. Diabetes Care. 2004; 27: 1487-1495.

37. Diabetes Prevention Program Research Group. Reduction in the incidence of type 2 diabetes with lifestyle intervention or metformin. N Engl J Med. 2002; 346 (6): 393-403.

38. Ely E, Gruss S, Luman E, Gregg E, Ali M, Nhim K et al. A national effort to prevent type 2 diabetes: participant-level evaluation of CDC's National Diabetes Prevention Program. Diabetes Care. 2017; 40 (10): 1331-1341.

39. Medina C, Jáuregui A, Campos I, Barquera S. Prevalencia y tendencias de actividad física en niños y adolescentes: Resultados de ENSANUT 2012 y ENSANUT MC 2016. Salud Pública de México. 2018; 60: 263-271.

40. Mauro I, López S, Garicano E, García B, Blumenfeld J. Detección de la alteración del metabolismo gluídico y resistencia a la insulina en una muestra piloto infantil: Aproximación metabolómica. Universidad y Salud. 2019; 21 (3): 191-197. 